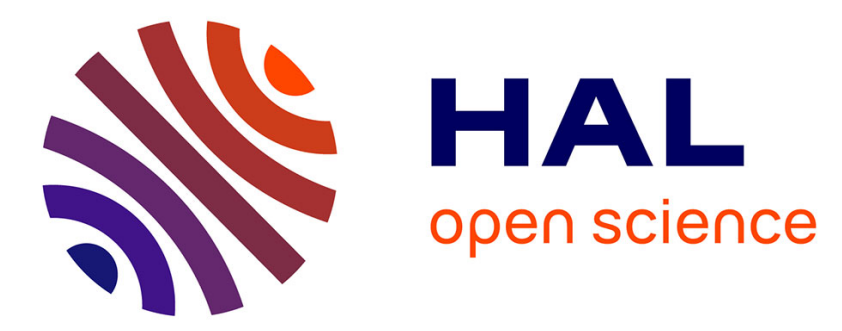

\title{
L'articulation des données en première et troisième personnes. De la genèse d'une méthodologie originale en Science du sport
}

Matthieu Quidu, Brice Favier-Ambrosini

\section{- To cite this version:}

Matthieu Quidu, Brice Favier-Ambrosini. L'articulation des données en première et troisième personnes. De la genèse d'une méthodologie originale en Science du sport. Intellectica - La revue de l'Association pour la Recherche sur les sciences de la Cognition (ARCo), 2014. hal-01756735

\section{HAL Id: hal-01756735 \\ https://hal.science/hal-01756735}

Submitted on 2 Apr 2018

HAL is a multi-disciplinary open access archive for the deposit and dissemination of scientific research documents, whether they are published or not. The documents may come from teaching and research institutions in France or abroad, or from public or private research centers.
L'archive ouverte pluridisciplinaire HAL, est destinée au dépôt et à la diffusion de documents scientifiques de niveau recherche, publiés ou non, émanant des établissements d'enseignement et de recherche français ou étrangers, des laboratoires publics ou privés. 


\title{
L'articulation des données en première et troisième personnes.
}

De la genèse d'une méthodologie originale en Science du sport

\author{
Matthieu QUIDU** \& Brice FAVIER-AMBROSINI"
}

\begin{abstract}
RÉSUMÉ. Classiquement étudiées depuis des méthodologies et des programmes de recherche cloisonnés, fonctionnant indépendamment, les données en première personne (documentant l'expérience vécue de l'acteur depuis son propre point de vue) et en troisième personne (objectivant les dimensions extérieures, publiques des conduites) sont, depuis une décennie, articulées dans l'optique d'accéder à une vision plus globale et complexe des actions. Comment le champ des Sciences du sport a-t-il contribué à la diffusion de cette méthodologie originale consistant en la confrontation de matériaux hétérogènes ? Notre analyse épistémologique examine les conditions, sociales et épistémiques, de sa genèse (conquête progressive d'objets diversifiés, référence à des travaux exemplars, dissémination à partir d'un noyau dur d'auteurs...) jusqu'à l'instauration d'une activité se rapprochant de la science normale. Elle formalise également la diversité des modalités d'articulation entre ces données (corrélation, discordance heuristique...) avant d'évaluer les effets de connaissance spécifiquement générés (renforcement de la robustesse par triangulation, découverte de nouvelles régularités, transformation des pratiques d'intervention...). Au final, l'articulation des points de vue en première et troisième personnes constitue un exemple en acte d'une pratique authentiquement interdisciplinaire.
\end{abstract}

Mots-clés : Cours d'action, cours d'in-formation, anthropologie cognitive, autoconfrontation, STAPS, épistémologie, pratiques méthodologiques.

ABstract. Joint Analysis of First and Third Persons Data. Genesis of an Original Methodology in Sport Sciences. Classically studied from methodologies and partitioned research programs operating independently, first person data (documenting actor's experience from his own point of view) and third person data (documenting external and public dimensions of behaviors) are, for a decade, articulated in order to access to a more complex, global and unitary view of acts. How did the Sport Science field contribute to the spread of this original methodology consisting in the confrontation of heterogeneous materials? This epistemological paper studies socials and epistemic requirements from its genesis (progressive conquest of diversified objects, reference to exemplars works, strewing of surveys from a hard core of authors...) to the establishment of a new normal science activity.

\footnotetext{
* Laboratoire d'histoire des sciences et de philosophie (UMR 7117 CNRS-Nancy Université) - Centre de recherche et d'innovation sur le sport (EA 647, Université Lyon I)-Laboratoire Activité, Connaissance, Transmission, Éducation (EA 4281, Université Clermont-Ferrand). quidumatthieu<at>yahoo.fr.

\# Laboratoire Analyse Comparée des Pouvoirs (EA 3350, Université Paris-Est Marne-la-Vallée) Groupe de recherche en Épistémologie, Histoire et Sociologie du sport. brice.favier<at>voila.fr.
}

(C) 2014 Association pour la Recherche Cognitive. 
It also formalizes the diversity of joints between all data (correlations, discrepancies...), before appraising the specifically generated knowledge effects (robustness strengthening by triangulation, discovery of new patterns, processing of intervention practices...). Ultimately, articulation between first and third persons data is an example of genuinely interdisciplinary practice.

Keywords : Course of action, course of "in-formation", cognitive anthropology, selfconfrontation interview, Sport Sciences, epistemology, methodological issues.

\section{I - INTRODUCTION}

Depuis près d'une décennie, un ensemble de publications partagent l'ambition d'articuler des matériaux empiriques de natures distinctes que nous qualifierons, à la suite de Varela (1996), de données respectivement en première et en troisième personnes. La présente analyse épistémologique étudie le développement de cette méthodologie originale dans le domaine des Sciences du sport ${ }^{1}$. Celui-ci a notamment généré les premières opérationnalisations empiriques de l'objet théorique du «cours d'information », proposé par Theureau (2009) dans le cadre du programme du Cours d'action ${ }^{2}$ (Sève et al., 2012). Plus précisément, il s'agira d'envisager les conditions, épistémiques et sociales (Berthelot, 1990), de la diffusion de cette méthodologie, de formaliser les diverses modalités effectives d'articulation entre matériaux hétérogènes et d'apprécier les effets spécifiques de connaissance produits.

Les données en troisième personne constituent les matériaux objectivables classiques des sciences expérimentales; elles correspondent aux informations qui peuvent être obtenues depuis un observateur extérieur, indépendamment de ce que vit l'acteur. Le codeur, non impliqué dans la situation, cherche à documenter la facette publique, visible, ostensible, en un mot comportementale des conduites. Sont ici incluses des mesures, «non basées sur le point de vue du sujet agissant » (Vermersch, 2010, p. 26) qui peuvent être «captées » ou «enregistrées » comme les signaux neuro-électriques, les variables physiologiques, les paramètres biomécaniques... L'adoption de ce type de données se fonde tantôt sur une nécessité lorsque le sujet est incapable de verbaliser son propre vécu ou que le mécanisme étudié est jugé a priori non accessible à la conscience tantôt sur une conviction lorsque le chercheur considère que les indicateurs comportementaux suffisent pour atteindre le but recherché et que le point de vue du sujet ne peut être considéré comme fiable. Ce type de matériaux jouit d'une puissante légitimité épistémique pour une science qui vise la production de connaissances objectivables se voulant indépendantes de l'observateur (Quidu, 2014).

Il n'en va pas de même pour les données dites en première personne, longtemps jugées comme «non scientifiques», car «trop difficiles à

\footnotetext{
${ }^{1}$ Ou Sciences et Techniques des Activités Physiques et Sportives (STAPS), $74{ }^{\text {ème }}$ section du Conseil National des Universités. Il n'est pas ici question de débattre de l'identité socio-épistémique des STAPS (Terral, 2003).

${ }^{2}$ Nous utiliserons la forme majuscule « Cours d'action » pour évoquer le programme dans sa généralité et la forme minuscule « cours d'action » pour évoquer l'objet théorique dans sa spécificité.
} 
quantifier $»^{3}$ (Vermersch, 2010, p. 26). Celles-ci visent à renseigner les facettes intimes, privées, ressenties, subjectives, singulières des actions. On s'intéresse ici à l'expérience vécue par le sujet selon son propre point de vue. Le sujet accède au statut d' « informateur principal » qu'il soit aidé ou non par un tiers pour accéder à son propre vécu4. Nous suivrons Vermersch (2010) lorsque ce dernier restreint l'acception des «données en première personne » aux "explicitations descriptives d'un vécu singulier de référence associé à une action bien délimitée ». Ce faisant, nous exclurons de cette catégorie les réponses différentielles (perception ou non d'un stimulus; score à une échelle d'auto-évaluation...) et les verbalisations du sujet jugeant son action ${ }^{5}$. Ces deux types de matériaux ne produisent pas à proprement parler une compréhension de l'expérience vécue dans la mesure où le sujet est sommé de se positionner par rapport à des catégories pré-codées, a priori, préexistant à l'enquête. À l'inverse, l'enjeu des données en première personne, d'inspiration phénoménologique, consiste à accéder à ce qui fait sens pour l'acteur, à ce qui «lui apparaît, sans la réfutation préalable de l'objectivité ou de la validité extrinsèque » (Gouju, Vermersch \& Bouthier, 2003). Les contenus des catégories de l'expérience sont à « remplir » par le sujet lui-même, d'une façon ouverte et indéterminée.

Ce projet de rendre compte du point de vue du sujet relativement à sa propre expérience s'ancre sur la volonté théorique de dépasser « ce qui résiste à l'analyse externe, à la description d'un tiers », c'est-à-dire les limites du point de vue en troisième personne qui, «s'il permet de gagner en rigueur apparente, risque fort de perdre la personne» (Vermersch, 2010, p. 26). Le recueil de matériaux en première personne jouit, dans les représentations collectives,

\footnotetext{
${ }^{3}$ Notons qu'il est impossible d'amalgamer d'une part un type de données (en première ou troisième personne) et d'autre part un type de traitement (qualitatif ou quantitatif). En effet, si la plupart des données en première personne subissent une approche de type qualitatif, il n'en va pas toujours ainsi. C'est le cas chez Bourbousson et al. (2014) qui, étudiant l'activité collective en basket-ball, suggèrent d'appliquer à des données d'expérience issues d'entretiens d'auto-confrontation des outils de quantification inspirés de la Social network analysis, habituellement mobilisés pour traiter des matériaux objectivables.

${ }^{4}$ Sont ici distinguées des données dites «radicalement en première personne » lorsque l'acteurinformateur n'est pas aidé par un tiers, des données dites "en première personne dans un sens faible » (ou en «seconde personne») lorsque la description de l'acteur est médiée par le chercheur. Dans le cadre de la présente réflexion, ces deux types de données seront inclus sous l'appellation, au sens large, «données en première personne »: dans les deux cas, c'est le point de vue du sujet agissant qui est visé. ${ }^{5}$ Seront ainsi exclues de notre corpus des recherches menées en Sciences du sport articulant d'un côté des données objectivables et de l'autre des données issues d'échelles de perception ou de questionnaires. D'une certaine façon, ces travaux peuvent être lus comme des prolongements des perspectives ouvertes en psychologie cognitive par Ericsson \& Simon (1980) qui confrontent des comptes-rendus verbaux et des matériaux comportementaux. Voir par exemple les travaux de Chalabaev $(2006,2009)$ confrontant, dans le cadre de l'étude de l'influence des stéréotypes sexués sur la motivation sportive, des marqueurs physiologiques et des échelles d'auto-évaluation de l'anxiété. Voir aussi Vanhelst et al. (2012) qui comparent deux méthodes d'évaluation de l'activité physique, par questionnaire auto-rapporté et accélérométrie. Le groupe d'études, articulant des données issues de questionnaires et d'enregistrements objectivables (le plus souvent des mesures physiologiques), apparaît comme majoritaire, d'un point de vue quantitatif, relativement aux travaux articulatoires recourant aux matériaux phénoménologiques; et ce tant dans le domaine des Sciences du sport que dans la littérature internationale en Sciences cognitives.
} 
d'une légitimité épistémique controversée. Il suscite doute et méfiance qu'illustre Lahire (1999, p. 46) incitant à «se méfier de la phénoménologie spontanée des acteurs » et évoquant «les ravages que peuvent produire les démarches dites parfois compréhensives ou subjectivistes, qui en restent à ce que les gens disent spontanément de ce qu'ils font $»$.

Face à ce scepticisme, les chercheurs visant à documenter le point de vue vécu par le sujet développent une critique sophistiquée des sources, soutenant que l'accès à l'expérience subjective n'est ni direct, ni spontané, ni transparent mais s'opère sous conditions. Par exemple, pour Mouchet (2003, p. 194), « la verbalisation de l'action se heurte à plusieurs problèmes difficiles : l'action est en grande partie une connaissance autonome contenant une part cruciale de savoir-faire-en-acte, c'est à dire non conscients et implicites; verbaliser une action n'est pas habituel, nous n'avons jamais été formés à le faire; les techniques efficaces pour aider à la verbalisation sont largement contreintuitives ». Une réflexion est également menée sur les critères de validité des données de verbalisation, que celle-ci soit intrinsèque $e^{6}$ ou extrinsèque : dans ce second cas, le point de vue de l'acteur est-il compatible avec l'objectivation que pourrait donner de son action un observateur extérieur indépendant? On entrevoit ici une première forme d'articulation entre données en première et en troisième personnes.

D'une certaine façon, l'ascèse méthodologique consistant à articuler des données de nature différente n'a rien de radicalement novatrice; elle constitue même une traduction spécifique du principe de «triangulation » des données (Van Der Maren, 1996) : un résultat est en effet d'autant plus robuste qu'il peut être dérivé depuis plusieurs méthodes indépendantes (Wimsatt, 1981). Toutefois, depuis une décennie, un ensemble de publications dans le domaine des Sciences du sport ont tenté de dépasser ce premier niveau d'articulation des données en première et troisième personnes afin, non seulement de renforcer la validité des résultats produits, mais surtout d'accéder à la dimension complexe et globale des actions sportives.

Comment ce champ, qui a classiquement étudié l'activité motrice depuis des points de vue développés indépendamment, en est-il venu à travailler leur articulation? Quelles sont les conditions, sociales, épistémiques et axiologiques, de possibilité de la genèse puis de la diffusion de cette méthodologie originale? Quelles en sont les modalités effectives de mise en œuvre ? Pour quels effets différentiels de connaissance ?

L'analyse des conditions, modalités et effets de connaissance associés au développement de la méthode originale d'articulation des données hétéroclites a supposé la constitution d'un corpus empirique en Sciences du sport. Ce dernier sera qualifié de «corpus occasionnel provoqué » (Quidu, 2011a, p. 69) : occasionnel tout d'abord par opposition à systématique : en effet, il ne s'est pas agi d'étudier exhaustivement les textes émanant de certaines revues ou de certains chercheurs en STAPS mais bien de n'en retenir que ceux plaçant explicitement au cœur de leur démarche la problématique de l'articulation des données hétérogènes; provoqué ensuite par opposition à accidentel, car la

\footnotetext{
${ }^{6}$ Voir par exemple l'adoption d'une «position de parole incarnée » dans le cadre de la psychophénoménologie et de la pratique de l'entretien d'explicitation (Petitmengin, 2005 ; Depraz, 2004).
} 
recherche de textes a été intentionnelle; cette traque s'est réalisée en prenant appui sur les bibliographies des articles précédemment analysés (réseaux de renvois intertextuels), au travers d'une activité de veille scientifique prolongée et via une recherche méthodique ${ }^{7}$ sur diverses bases de données, francophones et anglophones, généralistes et spécialisées, dans le domaine des Sciences du sport.

Au final, nous avons constitué un corpus composé d'une quarantaine de publications en Sciences du sport, de 2001 (date de parution de l'article princeps de Roberts) à 2014 (voir Annexe I). Celui-ci inclut principalement des articles issus de revues internationales à comité de lecture, mais également des actes de colloques et des manuscrits de thèse. Certains textes, publiés dans des revues de renom par des chercheurs expérimentés, ont reçu une audience importante et sont fréquemment cités par leurs pairs quand d'autres, publiés par des chercheurs novices, sont demeurés plus confidentiels, notamment du fait de leur nature (mémoires universitaires). Le choix a été fait d'inclure ces deux types de textes dans le corpus. Ce faisant, il nous sera possible d'apprécier la vitalité et le dynamisme d'un programme de recherche, envisagé dans son développement historique et son procès de ramification. Ce dernier se compose certes de textes fondateurs, de «travaux exemplars» (Soler, 2014), mais aussi de contributions plus anonymes, qui permettent toutefois d'opérationnaliser les ambitions théoriques, ontologiques et méthodologiques du programme tout en conquérant de nouveaux objets empiriques.

Sur ces corpus, ont été appliquées trois approches épistémologiques respectivement qualifiées de «compréhensive», d' «analytique» et de «normative » (Quidu, 2011a). La première approche, compréhensive, cerne les mobiles et conditions de possibilité, tant épistémiques, sociaux qu'ontologiques, du développement contemporain d'une telle démarche (voir II). La deuxième approche, analytique, (Berthelot, 1990), consiste à décrire puis à formaliser l'architecture logique des productions scientifiques afin d'en abstraire le mode spécifique ${ }^{8}$, déployé in acto, d'articulation entre données hétérogènes (voir III). L'ambition est d'englober, dans une typologie, la totalité des stratégies effectives d'articulation, jusqu'à l'atteinte d'un degré satisfaisant de saturation empirique. Enfin, la troisième lecture, normative, apprécie les effets différentiels de connaissance produits par l'articulation premièretroisième personnes, notamment comparativement aux apports épistémiques que peuvent générer des travaux travaillant séparément ces deux points de vue (voir IV).

Ces trois types d'approches ont été menés individuellement par les deux auteurs, puis confrontés dans l'idée de renforcer la robustesse des analyses via

\footnotetext{
${ }^{7}$ Les mots-clés utilisés pour la recherche sur bases de données (CAIRN, PERSEE, INIST, $S P O R T$ 'docs...) ont été : «première personne, troisième personne, articulation première-troisième personnes, sport, Sciences du sport, mouvement, motricité »...

${ }^{8}$ Plus précisément, il s'est agi d'identifier les types de données articulées, l'éventuelle antériorité d'une catégorie de données, le degré d'équilibre épistémique, le mode d'articulation effectivement mis en œuvre (corrélation, détermination causale...), son appui sur des concepts articulatoires. Un va-et-vient, dans le principe d'une grounded theory (Glaser \& Strauss, 1995), a été effectué entre d'un côté des études de cas concrets et de l'autre des efforts de construction d'idéals-types pour formaliser des modes génériques de confrontation.
} 
une triangulation. En cas de lectures divergentes d'une même production scientifique, les codeurs indépendants confrontaient leurs arguments avant de s'accorder sur une interprétation unique.

\section{II - DE LA DIFFUSION D'UNE MÉTHODOLOGIE ORIGINALE EN SCIENCES DU SPORT : CONDITIONS ÉPISTÉMIQUES ET SOCIALES D'UNE GENÈSE}

\section{II.1 - Conquête progressive d'objets diversifiés}

Les chercheurs en Sciences du sport proposant d'articuler les matériaux en première et troisième personnes ont conquis progressivement, depuis une dizaine d'années, un ensemble de thématiques de recherche.

Un premier objet privilégié a consisté à étudier la part de l'activité humaine préréflexive et difficilement verbalisable, non spontanément accessible à la conscience réflexive. À ce titre, est particulièrement investiguée la

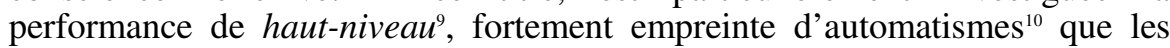
données en première personne, seules, ne suffisent à documenter. Dès lors, l'articulation des données permet de rendre compte de l'activité dans sa globalité complexe, de sa part verbalisable-conscientisable comme de sa frange infra-consciente. Comme le précisent Gal-Petitfaux et al. (2013, p. 263), « une part importante des actions motrices des athlètes de haut-niveau est fortement automatisée, au point que leurs propriétés biomécaniques reposent parfois sur des processus non accessibles à la conscience préréflexive de l'acteur et non verbalisables par lui ». Ce que corroborent Saury et al. (2010, p. 5) : «en aviron, une partie importante des ajustements inter-rameurs repose sur des processus non intentionnels et implicites ».

Une deuxième «situation d'étude privilégiée » (Grison et Riff, 2002) par l'articulation concerne les activités à médiation instrumentale ou comportant une interaction avec un dispositif technologique (aviron, natation avec le MAD-system, golf, escalade sur glace...). Evoquant les travaux de Roberts (2001, 2006) sur le matériel de golf, Gal-Petitfaux et al. (2013, p. 260) indiquent: " ces études ont montré l'incomplétude des données biomécaniques pour comprendre l'activité sportive instrumentée d'athlètes de haut-niveau, et ont souligné l'apport complémentaire de données relatives à la perception subjective qu'en ont les athlètes ».

Sont également travaillés les processus interpersonnels de communication, notamment autour de deux directions :

- l'étude des processus émotionnels de communication (Poizat, 2006; Martinent et al., 2014; Visioli, 2009): si l'émotion se définit comme une «forme de communication et de coordination» (Dumouchel, 1999), il convient tout à la fois de s'intéresser à ce que l'acteur ressent, à ce qu'il donne à voir corporellement et au degré de convergences-divergences entre ces deux facettes. Visioli (2009), étudiant l'expertise enseignante en Éducation physique

\footnotetext{
${ }^{9}$ L'expertise est parfois étudiée par contraste avec des pratiquants novices, comme c'est le cas chez Seifert et al. (2014).

${ }^{10}$ Et ce d'autant plus que l'habileté est cyclique, comme dans le cas de la natation (Gal-Petitfaux et al., 2013) ou de l'aviron (Saury et al., 2010).
} 
et sportive (EPS) du point de vue de la théâtralisation des émotions, confronte ainsi des données issues d'entretiens d'auto-confrontation (que ressent l'acteur?) et d'une analyse de la communication non verbale (qu'exprime-til ?).

- l'étude des processus interpersonnels de coordination (Bourbousson et al., 2014 ; Trohel, 2005; Saury et al., 2010). Étudiant la dynamique de construction de l'activité collective en basket-ball, Bourbousson \& Fortes (2012, p. 179) articulent les cadres théoriques du Cours d'action (matériaux en première personne permettant d'apprécier l'évolution du partage des significations entre joueurs) et de l'approche dynamique des systèmes non linéaires (matériaux en troisième personne permettant de «pointer les dynamiques de stabilisation-déstabilisation des couplages entre joueurs »).

\section{II.2 - Travaux exemplars (Soler, 2014)}

L'analyse met en lumière plusieurs contributions exemplars pour les Sciences du sport, c'est-à-dire fonctionnant «comme des modèles » pour les études ultérieures et participant à la diffusion de cette méthodologie. À l'échelle internationale, les travaux en langue anglaise de Roberts $(2001,2006)$ constituent des articles de référence pour les chercheurs français qui en retiennent le caractère pionnier quant à l'idée d'une articulation des données expérientielles et objectivables «afin d'en pointer les convergences ou divergences » (Seifert, 2010, p. 105). Au niveau francophone, la référence aux contributions «paradigmatiques » (Kuhn, 1983 ; Berthelot, 2001) ne sert plus simplement à légitimer le principe d'un croisement de données hétérogènes. Ces contributions sont considérées comme des modèles concrets, en acte, d'articulation qu'il convient de prolonger. Il en va ainsi de la recherche de Poizat (2006) citée par Bourbousson \& Fortes (2012, p. 179) : «Poizat (2006) a mobilisé les outils de l'ethnométhodologie pour appréhender finement les interactions dans un double de tennis de table. Il pointe des structures d'interactions récurrentes, non perçues par les sportifs eux-mêmes, et renseigne ainsi l'articulation des cours d'in-formation des pongistes». L'auteur est considéré par Saury et al. (2010, p. 22) comme ayant effectué « un premier pas vers l'objet théorique du cours d'in-formation ${ }^{11}$, en révélant des dimensions de la culture des pongistes incorporées dans leur activité en compétition». L'article de Saury et al. (2010) s'installe également comme un texte remarquable dans l'histoire récente de cette méthodologie, notamment de par sa mobilisation de données mécaniques. Gal-Petitfaux et al. (2013, p. 260), eux-mêmes cités par Seifert et al. (2014), s'inscrivent dans sa continuité et le considèrent comme «un exemple princeps » de travail visant à «décrire les processus de coordination motrice entre rameurs conjointement aux niveaux où l'activité est signifiante et non signifiante pour les acteurs ».

Enfin, l'article de Bourbousson \& Fortes (2012) tend à s'ériger comme une référence incontournable sur le plan épistémologique, les auteurs portant la discussion sur les conditions et modalités possibles d'articulation entre

${ }^{11}$ La définition de cet objet théorique est développée dans la section III.4. De façon succincte, le cours d'in-formation reconstitue la dynamique du couplage structurel de l'acteur à son environnement, que les éléments impliqués soient significatifs ou non pour l'acteur. 
matériaux hétérogènes. Leur thèse d'une articulation «faible », c'est-à-dire s'effectuant uniquement au niveau des résultats empiriques et sans concession ontologique, entre anthropologie cognitive située et approche dynamique, est notamment reprise par Seifert (2010) ou Martinent et al. (2014).

$\mathrm{Au}$ final, la référence fréquente à des travaux paradigmatiques, conjuguée à la conquête progressive d'objets variés, atteste de l'installation d'une activité tendant vers la «science normale », où prévaut la pensée «convergente » (Kuhn, 1983), moins de dix ans après les premières publications articulatoires qui se voulaient, quant à elles, critiques ${ }^{12}$. Reconnaître que, d'un point de vue qualitatif, ces développements présentent certains traits caractéristiques d'une science normale ne présage en rien, d'un point de vue quantitatif, de la diffusion massive de cette approche, dans le cadre du programme du Cours d'action, dans le paysage des Sciences du sport ou dans le champ académique général de la psychologie et de l'anthropologie cognitive (les données phénoménologiques y demeurent encore majoritairement suspectes). À ces trois niveaux, les opérationnalisations empiriques des objets théoriques du cours d'action ou du cours d'in-formation ainsi que les quelques tentatives élaborées depuis le cadre psycho-phénoménologique, demeurent, bien qu'en augmentation, rares, éparses, minoritaires si ce n'est marginales et contestée.

\section{II.3 - Réseau de diffusion}

La méthodologie d'articulation mise en œuvre en Sciences du sport s'organise principalement autour d'un «noyau central» d'auteurs engagés dans le programme de l'anthropologie cognitive située. Celui-ci est composé de Jacques Saury, de Carole Sève et de Jérôme Bourbousson, implantés institutionnellement à l'Université de Nantes ainsi que de Germain Poizat, désormais en poste à l'Université de Genève. Un second noyau existe également à l'Université de Clermont-Ferrand où enseignent actuellement Michel Récopé et Nathalie Gal-Petitfaux et où a exercé Luc Ria qui y a dirigé la thèse de Jérôme Visioli. Certains chercheurs, comme Alain Mouchet (Université de Paris-Est-Créteil), apparaissent, pour leur part, plus isolés géographiquement et institutionnellement dans leurs productions articulatoires.

Se citant mutuellement, les auteurs du noyau central constituent autour d'eux un réseau de chercheurs, a priori extérieurs au programme du Cours d'action mais spécialistes de l'étude extrinsèque du comportement, avec lesquels ils cosignent leurs articles. Ces collaborations sont d'une certaine façon imposées par l'objet théorique du cours d'in-formation qui, prenant acte des limites associées au cours d'expérience ${ }^{13}$ ne donnant accès qu'au niveau significatif pour l'acteur, incite à multiplier les observatoires et à confronter les

\footnotetext{
${ }^{12}$ Et donc potentiellement révolutionnaires au sens de Kuhn, c'est-à-dire à l'origine d'une crise paradigmatique, d'une remise en cause des postulats fondateurs de l'ancienne science normale. Celle-ci était en l'occurrence fondée sur un modèle de la « commande » et de la planification, en opposition avec les principes de l'émergence, de l' « autonomie » et de l'auto-organisation (Durand \& Arzel, 2002).

${ }^{13}$ Le cours d'expérience est une « réduction de l'activité à sa partie qui est significative pour l'acteur » (Saury et al., 2010, p. 4). Il décrit « l'activité d'un acteur engagé dans une situation, qui est significative pour ce dernier, c'est-à-dire montrable, racontable et commentable par lui à tout instant, moyennant des conditions favorables » (Theureau, 2004, p. 48).
} 
matériaux en première personne, conférant le primat à l'intrinsèque, et les données en troisième personne. Celles-ci peuvent être, suivant les études, de types biomécanique (Gal-Petitfaux et al., 2013 ; Poizat et al., 2011, Saury et al. 2010 ; Seifert et al., 2014) ; physiologique (Berteloot, 2008) ; conversationnel (Trohel, 2005); comportemental (Bourbousson \& Fortes, 2012). Ainsi, Antoine Nordez, spécialiste nantais de biomécanique (Nordez, 2006) cosigne en 2010 un article sur la coordination interpersonnelle en aviron avec Saury et Sève; de son côté, Marina Fortes, spécialiste nantaise des systèmes dynamiques (Fortes, 2003), cosigne en 2012 avec Bourbousson une étude sur la dynamique collective en basket-ball ; enfin, Ludovic Seifert, spécialiste rouennais ${ }^{14}$ de biomécanique et de l'approche dynamique du contrôle moteur (Seifert, 2010), coécrit avec Gal-Petitfaux, Adé et Poizat en 2013 un article sur l'activité propulsive en natation et en 2014 sur l'activité d'escalade sur glace.

Le noyau dur participe enfin à la diffusion de la méthodologie articulatoire au travers de l'encadrement de travaux universitaires : Carole Sève a encadré, en plus de la thèse de Germain Poizat, celle de Jérôme Bourbousson; de son côté, Jacques Saury a dirigé le master d'Anne Berteloot et la thèse de Jean Trohel qui a lui-même suivi par la suite Justine Colin en master.

\section{II.4 - Profils des chercheurs articulatoires}

Les chercheurs développant des travaux articulatoires manifestent des préférences ontologiques ${ }^{15}$ singulières (Quidu, 2009). Celles-ci s'organisent notamment autour de deux grandes orientations convergeant vers le rejet d'une approche réductionniste, cloisonnée et, de ce fait, mutilante du réel :

- une sensibilité à la totalité, à la globalité, à l'unité : les travaux articulatoires s'ancrent sur le projet «d'étudier l'activité humaine dans sa globalité et son unité »(Gal-Petitfaux et al., 2013, p. 278). Ce désir se retrouve chez Berteloot (2008, p. 23) pour qui « la seule mesure des aspects objectifs de l'effort ne suffit pas à rendre compte de son aspect multiforme ». La perception de l'effort est alors définie, «non comme une simple perception», mais comme une «évaluation subjective complexe faisant intervenir la globalité de l'activité humaine » (p. 70). Enfin, Seifert (2010, p. 104) reconnaît que «ce qui a motivé [sa] démarche d'approche mixte est d'avoir accès à une compréhension du comportement qui ne se résume pas à une seule approche ». Dit autrement, « l'interaction de cadres théoriques, d'objets théoriques et/ou de méthodologies s'inscrit dans une vision systémique, où le tout dépasse la somme des éléments » (p. 104).

- une attirance pour la pluralité, la complémentarité, la complexité : pour Gal-Petitfaux et al. (2013, p. 278), «la prise en compte des différentes

\footnotetext{
${ }^{14}$ Carole Sève a été anciennement en poste à l'Université de Rouen où elle a d'ailleurs dirigé la thèse de doctorat de Germain Poizat.

${ }^{15}$ Voir le concept de thêmata chez Holton (1981). Ceux-ci sont définis comme des « conceptions premières de l'être », des "présupposés globalisants sur l'essence des phénomènes ». Ils permettent d'«étayer le travail scientifique en lui donnant un sens » (Berthelot, 1990). Le plus souvent défendus implicitement et en nombre relativement restreint, les thêmata se présentent sous la forme de couples d'opposition : élément versus totalité, unité versus diversité... Quidu (2009) a démontré le caractère affectif, existentiel, voire éthique, qui relie un chercheur à ses préférences thêmatiques.
} 
dimensions de l'activité des nageurs » permet de «comprendre la complexité des composantes par lesquelles les nageurs organisent leur activité de nage ». L'argumentaire est homologue chez Seifert (2010, p. 99) indiquant que son HDR «s'inscrit ontologiquement dans une approche considérant la motricité humaine comme un système complexe ${ }^{16}$, à l'interface entre la biomécanique, le contrôle moteur, l'approche des systèmes dynamiques». Ce rapport inclusif à la pluralité des paradigmes lui permet de «comprendre la complexité de la motricité humaine en dépassant une analyse purement mécanique du comportement». Partageant ce souci du dialogue, Mouchet (2012, p. 247) admet « réinvestir dans le métier de chercheur un mode de fonctionnement personnel plus général», caractérisé par «une attitude d'ouverture, de comparaison, un intérêt pour la complémentarité et l'enrichissement réciproque ».

$\mathrm{Ce}$ «mode de fonctionnement personnel » (i.e. attention à la totalité et la complexité des conduites couplée à une exploitation de la pluralité et de la complémentarité des approches théoriques pour satisfaire cette attention) qu'évoque Mouchet pourrait être lié à son expérience antérieure de professeur d'EPS 17 (Quidu, 2011). Celle-ci l'aurait sensibilisé à la globalité, à l'unité indissociable, à la multi-dimensionnalité des conduites ainsi qu'à leur grande variabilité..$^{18}$ En outre, Mouchet, à l'instar de la quasi-totalité des chercheurs articulatoires, a été lauréat de l'agrégation d'EPS, concours sensibilisant à la problématique de la diversité des approches théoriques et dont la préparation contribue à la stabilisation de dispositions métacognitives pluralistes (Quidu, 2012).

\section{III - MODALITÉS DE L'ARTICULATION}

\section{III.1 - Catégories de données}

Deux types de données en première personne sont classiquement utilisés par les travaux francophones en Sciences du sport. Le premier concerne les données issues de la description par l'acteur de son expérience vécue au niveau de la conscience pré-réfléchie ${ }^{19}$. S'inscrivant dans une perspective psychophénoménologique, ce type de comptes-rendus permet d'accéder à l'expérience subjective des sujets via l'entretien d'explicitation (Vermersch, 1994) : cette technique «consiste, grâce à un questionnement portant sur la structure de

\footnotetext{
${ }^{16}$ L'activité humaine est considérée comme un «système complexe » notamment du fait de la multiplicité et de la dynamique fluctuante des interactions (ou couplages) entre les éléments qui la composent.

${ }^{17}$ C'est également le cas chez Carole Sève, Jacques Saury, Nathalie Gal-Petitfaux, Michel Récopé, Luc Ria, Jérôme Bourbousson, Jérôme Visioli... Cette convergence dans les trajectoires est d'autant plus remarquable qu'en STAPS la proportion des enseignants-chercheurs anciennement professeurs d'EPS ne cesse de décroître (Collinet, 2003).

${ }^{18}$ Cette expérience a également aiguisé, chez ces chercheurs, le souci de l' « utilité pratique » de leurs travaux académiques (voir IV.5.).

${ }^{19}$ Ce niveau de conscience, non accessible à l'acteur sans un travail approfondi de conscientisation (ou de réfléchissement), est qualifié de «préréflexif » par le programme du Cours d'action (Theureau, 2010) et de «pré-réfléchi » (ou encore de « conscience directe» ou de « conscience en acte ») par le programme de la Psycho-phénoménologie (Vermersch, 2000).
} 
l'expérience sans induire de contenu, à amener le sujet interviewé à rejouer l'expérience plusieurs fois, tout en guidant à chaque passage son attention vers une facette différente de l'expérience et une maille temporelle de plus en plus fine » (Petitmengin, 2006, p. 87). Notons que les chercheurs en STAPS mobilisent finalement assez peu, à l'exception de Mouchet (2003), Burns (2013) ou Récopé (2007), ce type de matériaux. Ils privilégient à l'inverse ceux issus de l'entretien d'auto-confrontation. Il en va ainsi chez Gal-Petitfaux et al. (2013), Saury et al. (2010), Bourbousson \& Fortes (2012), Visioli (2009) ou Seifert et al. (2014).

S'inscrivant dans le programme du Cours d'action (Theureau, 2009), l'entretien d'auto-confrontation permet d'appréhender la dynamique de construction de significations par l'acteur, au cours de son interaction en situation. Pour ce faire, il est confronté à l'enregistrement audio-visuel ${ }^{20}$ de son activité et invité à expliciter, montrer et commenter les éléments significatifs pour lui, au niveau de la conscience préréflexive. Les relances de l'interviewer portent sur la description des sensations, perceptions, focalisations, préoccupations, émotions.

Trois catégories de données en troisième personne ont, à ce jour, été utilisées par les chercheurs articulatoires en Sciences du sport. Les données recueillies sur la base d'analyses mécaniques et biomécaniques représentent la première catégorie de matériaux extrinsèques. Par exemple, pour Saury et al. (2010, p. 7), «les données mécaniques ont été recueillies grâce au système Powerline, spécialement conçu pour réaliser des mesures embarquées en aviron ». Chez Gal-Petitfaux et al. (2013), le comportement des nageurs, enregistré pendant l'intégralité du protocole, «a fait l'objet de mesures de deux paramètres mécaniques essentiels à la propulsion dans l'eau: la fréquence gestuelle et la force palmaire appliquée sur les cales ». Seifert et al. (2014) enregistrent pour leur part les patterns de coordination segmentaire de grimpeurs sur glace afin de distinguer des coordinations en phase ou en antiphase.

Les données physiologiques constituent le deuxième type de données en troisième personne. Berteloot (2008) enregistre par exemple l'évolution de la fréquence cardiaque, mesure relativement facile à recueillir, durant une course de demi-fond avant de la corréler à la dynamique de perception de l'effort. Récopé et Filaire ${ }^{21}$, cités par Récopé (2007), ont quant à eux formulé le projet de recueillir le niveau de cortisol salivaire de volleyeurs, en tant qu'indicateur du stress précompétitif, avec l'hypothèse que les joueurs les plus sensibles à l'enjeu de la rupture présenteraient les taux hormonaux les plus élevés.

\footnotetext{
${ }^{20}$ Notons ici que l'entretien d'auto-confrontation contient, en son cœur même, un principe d'articulation de données en première personne (les verbalisations provoquées) et en troisième personne (les enregistrements audiovisuels). Celles-ci sont d'ailleurs synchronisées temporellement via la construction d'un «protocole à deux volets ». Les matériaux en troisième personne servent tout à la fois d'ancrage à l'acteur pour décrire son expérience vécue et de moyen d'évaluation de la vraisemblance des ressentis exprimés, de leur congruence voire de leur compatibilité aux évènements survenus (Poizat, Haradji \& Seifert, 2011).

${ }^{21}$ Les auteurs ont finalement abandonné ce projet d'articulation des données hétérogènes, lequel n'a pas fait l'objet d'une publication autonome. Cette interruption est un bon révélateur de la lourdeur, du coût comme de la complexité de ces protocoles méthodologiques, comme le reconnaît d'ailleurs Ria (2006).
} 
Enfin, une dernière catégorie, large, de données en troisième personne concerne la description extrinsèque des comportements moteurs ou non verbaux. Par exemple, Récopé (2007, p. 17-18) utilise plusieurs indicateurs comportementaux pour caractériser le degré de mobilisation défensive des volleyeurs : « orientation du corps par rapport au ballon ; attitude de garde ; engagement énergétique... ». De son côté, Visioli (2009) documente les modes non verbaux de communication au moyen d'une analyse proxémique (distances interpersonnelles, orientations du corps, expressions du regard...).

\section{III.2 - Modes typiques d'articulation}

Quatre modalités typiques d'articulation sont indentifiables en Sciences du sport.

Un premier mode typique d'articulation consiste en la mise en évidence de corrélations entre les deux types de données. Cette congruence renforce, de fait, la robustesse des résultats produits. Il en va ainsi chez Récopé (2007) dont les analyses comportementales et les matériaux d'explicitation convergent pour identifier les joueurs sensibles à l'enjeu de la rupture. Ainsi, «les comportements comme les propos du joueur mettent en évidence que sa mobilisation défensive est fondamentalement référée, à tout moment, à la rupture de l'échange» (p.97). Une telle convergence peut conduire à l'élimination d'un type de données afin d'éviter les redondances et de diminuer le coût d'une double méthodologie. Ainsi, Ria (2006), après avoir constaté une corrélation parfaite, chez les enseignants débutants, entre leurs scores à l'échelle d'auto-estimation des états affectifs et les variations de leur fréquence cardiaque, délaisse cette seconde technique en troisième personne (présence de validation externe).

Dans d'autres cas, au sein de la même étude, peuvent être mises en lumière tantôt des divergences tantôt des convergences entre données hétérogènes. Étudiant les processus décisionnels en rugby, Mouchet (2003) met parfois en lumière des caractéristiques communes entre les verbalisations provoquées et les comportements observés, c'est-à-dire entre ce que le joueur pense faire et ce qu'il fait effectivement: ainsi, «dans cette situation, une grande cohérence apparaît entre ses actions et ses verbalisations » (p. 250). Dans d'autres séquences, les divergences l'emportent: «nous avons plusieurs fois mis en évidence des différences entre les points de vue en première et en troisième personnes. C'est par exemple le cas d'un sujet qui déclare hésiter à faire une passe décisive en raison de la présence d'une main susceptible de gêner la passe. Or, cette main n'existe pas d'un point de vue externe» (p. 377). La structure argumentaire est homologue chez Berteloot (2008, p. 44), proposant une « représentation graphique, pour chacun des coureurs, de l'évolution de trois types de données (vitesse de course, fréquence cardiaque et perception de l'effort)» et y repérant «des évolutions simultanées ou au contraire différentes $» .^{22}$

\footnotetext{
${ }^{22}$ Voir aussi Visioli (2009) mettant en évidence des moments de couplage et de découplage entre le dire émotionnel (issu de l'auto-confrontation) et l'expression émotionnelle (observée lors de l'interaction).
} 
Dans une troisième modalité articulatoire que nous qualifierons de discordance heuristique, les deux types de données ne convergent pas, ce qui a pour conséquence de remettre en cause certaines hypothèses initiales et d'en formuler de nouvelles. S'intéressant à la perception de l'effort chez des coureurs de demi-fond, Berteloot (2008, p. 69) pointe « des discordances entre l'effort fourni objectif (vitesse de course et FC) et la perception de l'effort». Ceci remet dès lors en question « le lien de causalité directe qui existerait entre ces deux concepts, tel que le postulent les hypothèses de Borg ». De leur côté, Gal-Petitfaux et al. (2013, p. 279) enregistrent des résultats discordants sur le $M A D$-system, pour les vitesses moyennes, quant à la force objective exercée par les nageurs sur les cales et le ressenti subjectivement associé. Les auteurs concluent à une «appropriation» du dispositif, celui-ci devenant «transparent» pour l'utilisateur. Est ainsi exploitée «la portée heuristique des discordances mises à jour, apportant des enseignements cruciaux sur l'activité ».

Suivant un dernier mode dit du test d'hypothèses, l'analyse menée suivant un type de méthodologie révèle un problème à résoudre, lequel s'avère explicable par plusieurs hypothèses qui seront systématiquement testées par une analyse effectuée depuis l'autre méthodologie. Saury et al. (2010) exemplifient cette modalité dans leur étude des processus de coordination interpersonnelle en aviron. Les données en première personne, issues du cours d'expérience, révèlent un ressenti désagréable pour l'une des rameuses, une impression syncrétique «de ne pas être ensemble, d'être poussée par sa coéquipière, et de ne pas pouvoir réaliser pleinement son mouvement ». Or, «cette sensation peut être a priori liée à une diversité d'ajustements comportementaux dynamiques entre les rameuses, non seulement non significatifs pour elles, mais également trop subtils pour être identifiables, par observation ou analyse vidéo, par les entraîneurs eux-mêmes » (p. 21). Sont alors dégagées trois hypothèses relatives aux ajustements moteurs des rameuses susceptibles d'expliquer ces perceptions: a) décalage temporel à l'attaque du mouvement ; b) différence d'amplitude de rame ; c) différence de vitesse sur le retour aérien. L'analyse mécanique invalide finalement l'hypothèse a) démontrant que la «sensation de ne pas pouvoir réaliser pleinement son mouvement sous la pression de sa partenaire s'expliquerait principalement par un différentiel d'amplitude» et «serait amplifiée par un début de retour aérien plus lent » (p. 17).

\section{III.3 - Équilibre-déséquilibre des données}

Au-delà de la diversité des modes typiques d'articulation, les travaux articulatoires se différencient de par le degré d'équilibre statutaire et probatoire qu'ils accordent aux deux types de données.

Certaines contributions maintiennent un équilibre épistémique entre les données hétérogènes. C'est par exemple le cas chez Récopé (2007) documentant la mobilisation défensive des volleyeurs en combinant analyse extrinsèque des comportements moteurs et examen intrinsèque des ressentis affectifs. Ces deux types de données «travaillent» à égalité de statut et de valeur probatoire; ils sont considérés comme renseignant des dimensions complémentaires d'un même objet. Leur corrélation permet tout à la fois de 
renforcer la fiabilité des descriptions et leur complétude. Il en va de même chez Seifert et al. (2014) entre données issues de l'auto-confrontation afin de documenter la perception des affordances et données issues de l'analyse mécanique pour quantifier la variabilité des coordinations.

À l'inverse, d'autres travaux installent d'emblée, dans le procès d'articulation, une relation de subordination, non équitable, entre matériaux. Ce déséquilibre s'opère, dans le cadre du programme du Cours d'action, en faveur des matériaux expérientiels. Chez Visioli (2009), l'utilisation de données extrinsèques, issues de l'analyse des communications non verbales, ne remet en aucun cas en cause le primat de l'intrinsèque et l'hypothèse fondamentale de l'énaction (i.e. asymétrie des couplages de l'acteur à son milieu). Les matériaux objectivables n'offrent qu'un «complément au cadre principal » (p. 258) maintenu dans ses prérogatives. Dans la pratique de l'enquête, cette prévalence se traduit par l'antériorité de l'analyse des matériaux phénoménologiques. De celle-ci, vont être dégagées quelques séquences saillantes qu'il conviendra de soumettre à l'analyse proxémique. Dit autrement, il s'agit de «partir du point de vue de l'enseignant et de la tonalité émotionnelle de son expérience, puis de travailler à la mise en relation entre ce qui est dit et ce qui est montré » (p. 295). Ici, la confrontation des données en première et troisième personnes constitue, non pas une articulation de paradigmes concurrents, mais bien un dispositif méthodologique au service de la sophistication du programme du Cours d'action. Les présupposés épistémiques et ontologiques de ce dernier sont préservés sans contamination par ceux émanant des paradigmes alternatifs dont il se contente d'utiliser, en les réinterprétant, les outils méthodologiques. C'est en ce sens que Bourbousson \& Fortes (2014) parlent d'une articulation «faible », c'est-à-dire sans concession ontologique ${ }^{23}$.

\section{III.4 - L'articulation est-elle fondée conceptuellement?}

Suivant les publications, la confrontation des matériaux hétérogènes peut être soutenue théoriquement (au moyen de concepts unificateurs pensant spécifiquement la question de l'articulation) ou s'opérer de manière $a$ théorique par juxtaposition des données.

Lorsque les matériaux en première personne sont obtenus via entretien d'auto-confrontation par des chercheurs inscrits dans le programme de l'anthropologie cognitive située, le croisement des points de vue est englobé théoriquement grâce aux deux concepts (Bourbousson \& Fortes, 2012) :

- du «cours d'action»: cet objet théorique, mobilisé notamment par Berteloot (2008), Bourbousson et Fortes (2012) ou Trohel (2005) et défini par Theureau (2009), « inclut le cours d'expérience (i.e. qui restitue la dynamique de construction de significations par l'acteur suivant un primat de l'intrinsèque) auquel s'ajoute la description des contraintes ou effets

\footnotetext{
${ }^{23}$ Il semble toutefois plus évident d'emprunter les méthodes de programmes alternatifs possédant une certaine proximité voire connivence ontologique. C'est en tout cas ce que tend à indiquer Seifert (2010) répertoriant les convergences entre le programme du Cours d'action et l'approche dynamique non linéaire. Ceux deux cadres théoriques étudient, d'un point de vue historique, des systèmes complexes émergents comportant de nombreuses interactions.
} 
extrinsèques qui pèsent sur l'expérience d'un acteur » (Visioli, 2009, p. 179). Pour Bourbousson \& Fortes (2012, p. 177), « décrire les contraintes et effets extrinsèques du cours d'expérience d'un acteur [...] consiste à prendre en compte les éléments contextuels qui favorisent telle ou telle expérience de l'acteur, éléments qui en retour sont modifiés du fait même de l'expérience que l'acteur en fait ».

- du «cours d'in-formation»: partant du constat que, si le cours d'expérience permet d'appréhender les «caractéristiques significatives pour l'acteur », il laisse toutefois «dans l'ombre d'autres facettes de l'activité qui, tout en n'étant ni montrables, ni racontables, ni commentables, interviennent dans l'activité de l'acteur» (Saury et al., 2010, p. 22), Theureau (2009) propose l'objet théorique du cours d'in-formation. Celui-ci rend compte de la dynamique du couplage structurel, que les éléments qui le composent soient significatifs ou non pour l'acteur. Plus précisément, le cours d'in-formation « intègre des éléments ressortant du corps, de la situation et de la culture de l'acteur, qui ne donnent pas lieu à expérience, mais qui sont néanmoins pertinents pour l'organisation interne de son activité » (Saury et al., 2010, p. 22). La description du cours d'in-formation dépasse donc «les limites des descriptions du cours d'expérience de l'acteur dans la mesure où certains éléments n'ayant pas donné lieu à expérience peuvent être pris en compte ». Elle n'est cependant acceptable «dans une perspective énactive, qu'en accordant un primat au cours d'expérience, autrement dit, en se fondant sur une analyse préalable du cours d'expérience ». Par l'objet théorique du cours d'information, l'action est considérée comme un système complexe composé de multiples niveaux en interaction. Cet objet théorique est notamment mobilisé par Saury et al. (2010) ou Gal-Petitfaux et al. (2013).

Les objets théoriques du cours d'action puis du cours d'in-formation doivent être compris comme des développements successifs, au sein du programme du Cours d'action, dans l'optique d'intégrer des dimensions diversifiées de l'activité humaine et du couplage structurel (Sève et al., 2012). Là où le cours d'expérience ne s'intéresse qu'au niveau significatif pour l'acteur, à l' «effet de surface » du couplage structurel laissant dans l'ombre une large part de processus et de phénomènes «non conscientisables », le cours d'action intègre les effets et contraintes extrinsèques. Le cours d'information effectue un pas supplémentaire en considérant les dimensions non significatives pour l'acteur mais participant pourtant de son domaine cognitif.

À l'inverse, d'autres confrontations s'effectuent sur un mode a-théorique. Il en va ainsi chez Récopé (2007) ou Seifert et al. (2014) qui, bien qu'enregistrant de profondes convergences entre données en première et troisième personnes, ne s'avancent pas dans la théorisation de cette articulation. 


\section{IV - EFFETS DIFFÉRENTIELS DE CONNAISSANCE}

Quels sont les apports épistémiques spécifiquement générés par les efforts d'articulation ? À la suite de Lahire ${ }^{24}$ (1996), nous considérons que les effets d'une démarche (à un niveau méthodologique) ou d'un programme (à un niveau théorique) doivent être évalués comparativement, c'est-à-dire en les confrontant aux résultats qui ont été ou auraient pu être produits suivant un autre protocole. Qu'apporte de plus ou de différent ${ }^{25}$ une confrontation des points de vue par rapport aux travaux qui en proposent une étude cloisonnée ?

\section{IV.1 - La triangulation des données comme opérateur de renforcement de la robustesse des résultats}

Wimsatt (1981) définit la robustesse comme «l'utilisation de moyens multiples pour trianguler l'existence et les propriétés d'un phénomène ». Un résultat serait d'autant plus solide qu'il reste invariant sous plusieurs dérivations indépendantes, si et seulement si celles-ci peuvent être, chacune, considérées comme suffisamment valides intrinsèquement (Soler et al., 2012). C'est bien le croisement des techniques de collecte sur le même évènement qui en renforce la fiabilité en neutralisant l'incertitude (ou relativité) due aux instruments (Van Der Maren, 1996). Ria (2006) renforce la robustesse de sa description de la dynamique affective des enseignants débutants en triangulant données subjectives (auto-évaluation par le sujet du caractère confortable versus inconfortable de son vécu) et objectivables (enregistrement des variations de fréquence cardiaque). Ainsi, dans le cadre d'un moment désagréable, on enregistre simultanément ${ }^{26}$ une «accélération très forte du rythme cardiaque » et une «évaluation très négative de l'intensité des sentiments ».

\section{IV.2 - L'articulation des données comme dépassement des limitations intrinsèques à chaque programme élémentaire}

Selon Morin (1991), chaque paradigme comporte, en son noyau dur, une zone aveugle et une limitation intrinsèque auxquelles il ne peut accéder que par la médiation d'un autre système théorique. Dit autrement, l'exercice polémique suppose l'extériorité d'un regard autre $e^{27}$, jouant la double fonction de « décentration critique » et d' « objectivation réciproque » (Quidu, 2011a).

Pareille logique ${ }^{28}$ se retrouve dans le cadre de l'anthropologie cognitive qui noue des relations de complémentarité avec plusieurs programmes alternatifs. Par exemple, Bourbousson \& Fortes (2012) montrent qu'une collaboration

\footnotetext{
${ }^{24}$ Il est «scientifiquement fécond de prendre acte de la variation des effets de connaissance selon le contexte adopté »: «quels sont les gains et pertes explicatifs associés au passage d'un langage d'analyse à un autre ?».

${ }^{25}$ Poizat et al. (2012, p. 634) soutiennent à titre illustratif : «the results seemed to confirm that the combination of these data give a more comprehensive account of team coordination than each could do separately .

${ }^{26}$ Bühlmann \& Tettamanti (2007) parlent d'une situation de « convergence » de résultats.

${ }^{27}$ Pour Feyerabend (1979), « les préjugés sont mis en évidence par contraste, non par analyse ».

${ }^{28}$ Bühlmann \& Tettamanti (2007) parlent d'une situation de « complémentarité » des approches.
} 
Cours d'action-approche dynamique permet, dans le cadre de l'étude des coordinations interpersonnelles, de dépasser leurs limites respectives intrinsèques: d'un côté, l'anthropologie cognitive, en privilégiant la perspective de l'acteur notamment dans le cadre de l'objet du cours d'expérience, délaisse l'analyse de l'ensemble de l'activité et peut bénéficier de l'approche dynamique qui documente les contraintes et effets extrinsèques des cours d'expérience ; de l'autre côté, «l'approche dynamique, parce qu'elle appréhende le système à un niveau global d'analyse, se retrouve naturellement confrontée à la nécessité d'une approche interdisciplinaire lorsqu'il s'agit d'interpréter les comportements collectifs observés » (p. 180) ; elle peut, dès lors, profiter des analyses «à vocation plus psychologique » et compréhensive de l'anthropologie cognitive. D'autres auteurs (Visioli, 2009 ; Colin, 2009) tentent de dépasser la difficulté pour le Cours d'action à rendre compte de la dimension corporelle, incarnée, émotionnelle des interactions en mobilisant des programmes étudiant précisément les communications non verbales (analyses proxémiques). Dit trivialement, les travaux sur la «nouvelle communication » dégagent des formes d'interaction dont le Cours d'action documente la signification en situation.

Comme le résument Martinent et al. (2014, p. 302), les approches en première et troisième personnes semblent, chacune, «susceptibles d'explorer des processus ou mécanismes qu'il semble inenvisageable d'investiguer par l'intermédiaire d'une autre méthodologie ». Plus précisément, nous proposons d'interpréter la formalisation, dans le cadre de la dynamique intrinsèque de développement du programme du Cours d'action, de l'objet théorique du cours d'in-formation, comme un essai de dépassement des limites inhérentes à l'objet cours d'expérience dans la perspective d'approcher les dimensions globales et complexes des conduites et ce en multipliant les observatoires des actions humaines. Le cours d'expérience, en s'intéressant au domaine significatif pour l'acteur, achoppe en effet à rendre compte des mécanismes infra-conscients. Dans le cas de Saury et al. (2010), le dépassement de cette limite intrinsèque est permis par une convocation localisée des analyses mécaniques qui permettent d'accéder aux «automatismes et ajustements comportementaux subtils non significatifs pour les rameurs ». Il est ainsi possible de conférer une « signification mécanique » aux sensations syncrétiques.

\section{IV.3 - L'articulation comme moyen de découverte de nouvelles régularités}

Pour Seifert (2010, p. 104-105), «au-delà de l'apport de chaque cadre théorique, leur interaction fait émerger de nouvelles connaissances permettant de typicaliser l'activité humaine ». L'auteur formalise en effet, chez les nageurs confrontés à un dispositif d'évaluation de la performance propulsive, plusieurs «activités typiques » suivant les vitesses considérées. Ce lien entre «contexte de vitesse et perceptions des nageurs quant à l'appropriation d'un dispositif technologique sportif » serait «resté aveugle dans une démarche mono-disciplinaire » (Gal-Petitfaux et al, 2013, p. 275). Seifert et al. (2014) et Seifert (2010) distinguent pour leur part des activités typiques divergentes chez les experts et les novices en escalade sur glace du point de vue de la perception des affordances et de la variabilité des patterns de coordination. Par exemple, 
chez le grimpeur débutant, « le mode de coordination bras-jambes en phase, le grand nombre de frappes avant ancrage définitif et la faible confiance en lui lorsqu'il a des ancrages peu profonds conduisent à une forme d'activité-type «se construire des appuis de confiance» (Seifert, 2010, p. 114). À l'inverse, l'expert manifeste une plus grande flexibilité-une multi-stabilité-de coordination et une sensibilité perceptive accrue aux opportunités offertes par l'environnement.

De son côté, Mouchet (2003) met en évidence le compactage singulier et subjectif des diverses sources d'influence au sein du processus décisionnel du rugbyman. Plus précisément, il dégage des relations «fluctuantes» entre les circonstances singulières de la situation et l'arrière-plan décisionnel. Sont ainsi distinguées, suivant le degré de pression temporelle, des décisions de type «délibératoire» (i.e. «mise en rapport réfléchie d'un ensemble de données relatives à l'instant vécu et au passé ») et de type «en acte» (i.e. émergentes du couplage direct à la situation).

\section{IV.4 - L'instauration d'un réseau de "contraintes génératives mutuelles »(Peschard, 2004)}

Ici, la découverte d'une régularité sur un plan suscite la découverte de régularités sur l'autre plan ou tout du moins conduit à sophistiquer le recueil de matériaux sur cet autre plan. Illustratifs d'un tel réseau d'interpellations réciproques à un niveau méthodologique, Martinent et al. (2014, p. 303) suggèrent de mobiliser « les résultats de l'analyse à la troisième personne des comportements non verbaux » pour « modifier le guide d'entretien, support des entretiens d'auto-confrontation »: « les sportifs pourraient être spécifiquement amenés à explorer le caractère volontaire ou automatique de certains comportements non-verbaux ». Dans une même veine, Poizat et al. (2012, p. 638) considèrent que «la combinaison des données en première et troisième personnes est une voie intéressante pour le développement méthodologique dans la recherche sur les processus de coordination en sport $»^{29}$. Plus précisément, les auteurs proposent, dans la mesure où l'étude révèle que «certains comportements qui n'étaient pas spontanément décrits par les joueurs jouaient un rôle essentiel dans leur coordination $»^{30}$, d'encourager les joueurs à commenter ces comportements qui semblaient a priori non significatifs de leur point de vue : «une solution ${ }^{31}$ pourrait être de conduire l'entretien en deux parties: la première partie serait l'entretien d'autoconfrontation classique en vue de construire le cours d'expérience; la seconde partie serait dédiée à des commentaires d'évocation sur les détails de certains comportements qui n'avaient pas été spontanément décrits dans la première partie. Durant ce second visionnage de l'enregistrement vidéo, pourraient par exemple être utilisés les principes de l'entretien d'explicitation afin de faciliter

\footnotetext{
${ }^{29}$ «The combination of first- and third-person data is an interesting path for methodological development in research on coordination processes in sport».

${ }^{30}$ «Certain behaviours that were not spontaneously described by the players played an essential role in the coordination between them ».

${ }^{31} \mathrm{Qu}$ 'il reste à opérationnaliser empiriquement.
} 
des verbalisations plus détaillées au sujet de comportements relevés dans la première partie $\gg^{32}$ (p. 638).

À un niveau plus théorique, les données obtenues en troisième personne permettent d'affiner l'analyse du recueil en première personne jusqu'à y découvrir des nuances insoupçonnées : ainsi, toujours chez Poizat et al. (2012, p. 634), «l'analyse du cours d'expérience de chaque joueur confirme qu'à certains moments ces comportements typiques révélés par les descriptions en troisième personne étaient significatifs: les joueurs construisent des interprétations à propos de leur partenaire ou de la situation sur la base de leurs regards, mouvements et actions en lien avec la remise en jeu $\gg^{33}$.

\section{IV.5 - L'articulation des données hétérogènes au service de la transformation des pratiques}

Au-delà de ces apports épistémiques, l'articulation des points de vue comporte des effets praxiques ${ }^{34}$, ce qui confirme le souci de l'utilité sociale ${ }^{35}$ des chercheurs articulatoires. La transformation des pratiques peut tout d'abord concerner l'amélioration du matériel. Gal-Petitfaux et al. (2013, p. 276-277), à partir de l'étude de l'appropriation effective ${ }^{36}$ d'un dispositif d'évaluation en natation, suggèrent plusieurs «modifications de l'instrument de mesure ». À titre d'exemple, «il pourrait s'agir, pour les vitesses lentes et maximales, de changer la distance inter-cales » afin de se rapprocher des «situations de pratique naturelle». De cette façon, «le dispositif évaluerait au mieux ce pour quoi le concepteur l'a inventé : faire nager à différentes plages de vitesse pour établir une courbe Force-Vitesse ». La finalité ergonomique porte également sur la conception de nouveaux matériels instrumentant la pratique sportive. Il en va ainsi, chez Roberts (2001), pour la construction de clubs et de balles de

\footnotetext{
32 «One solution might be to conduct the interview in two parts: the first part would be the classic selfconfrontation interview to construct the course of experience; the second part would be dedicated to evoking comments on the details of certain behaviours that had not been spontaneously described in the first part. During this second viewing of the video recording, for example, the principles of the explicitation interview could be used to facilitate more detailed verbalizations about behaviours noted in the first part of the interview ».

${ }^{33}$ "The analysis of each player's course of experience confirmed that at certain moments these typical behaviours revealed by the third-person descriptions were meaningful: the players constructed interpretations about their partner or the situation on the basis of their partner's looks, movements, and actions linked to getting back into play ».

${ }^{34}$ Pour Visioli (2009, p. 157), il s'agit d'articuler « une visée épistémique et une visée transformative : décrire et comprendre l'activité des enseignants et contribuer à la conception d'aides à leur formation ». De son côté, Mouchet (2012, p. 246) souhaite dégager « des retombées possibles pour une intervention efficace et pertinente ».

${ }^{35}$ Plus précisément, les auteurs articulatoires aspirent au compromis optimal entre validité épistémique et utilité pratique. Ainsi, selon Mouchet (2012, p. 245), « tout chercheur est confronté au dilemme de la rigueur et de la pertinence (Schön, 1994). Il est possible de choisir l'un des deux pôles et c'est l'image qui est la plupart du temps associée aux sciences du vivant versus sciences humaines; ou de tenter de tenir les deux bouts comme nous le souhaitons. Le choix d'étudier les décisions en contexte réel de match et non en laboratoire, tout en élaborant un dispositif rigoureux de recueil et de traitement des données, s'inscrit dans ce compromis ».

${ }^{36}$ Qui a permis de mettre à jour «l'écart entre l'activité de nage prescrite par le dispositif et les adaptations gestuelles produites par les nageurs ».
} 
golf susceptibles d'améliorer les sensations ressenties lors du putting mais aussi chez Poizat et al. (2011) finalisant leur étude de l'activité des grimpeurs sur glace par la création de nouveaux piolets et crampons dont l'appropriation par le pratiquant serait facilitée.

Le second effet praxique a trait à l'optimisation des stratégies d'intervention. Les efforts d'articulation de matériaux hétérogènes portent sur différents objets d'acquisition. L'apprentissage est tout d'abord moteur lorsqu'il concerne l'incorporation de nouvelles coordinations motrices. À cet égard, Burns (2013) conçoit un dispositif d'entraînement par réalité virtuelle permettant l'acquisition d'une habileté complexe en karaté au moyen d'un avatar. De leur côté, Saury et al. (2010, p. 23-24) considèrent que la confrontation analytique des points de vue «constitue une aide pour les athlètes dans la compréhension et l'évaluation de la fiabilité de leurs propres sensations ». Un autre objet d'apprentissage envisagé a trait à l'optimisation des coordinations entre pratiquants au sein d'un même collectif. Dans ce cadre, Sève et al. (2013) considèrent que l'articulation des données peut faciliter « le partage d'expériences entre membres d'une équipe et entre entraîneurs et athlètes ; cela permet d'échanger les points de vue et les interprétations à propos du fonctionnement de l'équipe et des variations de performance $»^{38}$. Les divers protagonistes confrontent leur vécu subjectif respectif avec la performance objectivable du collectif. Peuvent alors être identifiées des convergences comme des divergences, entre les diverses expériences ou entre les expériences et les résultats produits. Mouchet (2014) envisage quant à lui de développer une «culture tactique commune » au sein de l'équipe de rugby. Il s'agit de faciliter la co-construction d'un référentiel commun, "interface entre le projet collectif élaboré en dehors de l'action et la décision individuelle ». Ce dernier «peut être enrichi et stabilisé en dehors de l'action par une analyse de pratique basée sur des entretiens permettant d'explorer le vécu subjectif ».

La transformation des pratiques pédagogiques s'opère également par la définition de nouveaux objectifs de travail. Chez Récopé (2007, p. 127), la finalité première d'une démarche efficace d'enseignement en volley-ball sera «l'incorporation par tous les pratiquants d'une sensibilité à l'enjeu de la rupture de l'échange »; cet objectif découle du constat que «les pratiquants défensivement mobilisés sont ceux qui s'avèrent systématiquement les plus performants en fin de séquence» (p. 128). Quant à Berteloot (2008), elle suggère de développer, chez les athlètes de fond, dès l'entraînement, l'«acceptation de l'effort» et ce en insistant sur la confiance et la connaissance de soi.

L'articulation des données en première et troisième personnes débouche enfin sur des propositions de renouvellement des dispositifs de formation des

\footnotetext{
${ }^{37}$ Burns a évalué l'efficacité différentielle de plusieurs techniques d'apprentissage moteur en considérant tout à la fois les progrès visibles évalués par un spécialiste de la discipline (troisième personne) et le processus d'acquisition décrit par l'apprenant lui-même (première personne).

${ }^{38}$ «...the sharing of experiences between crew members and between coaches and athletes, and it allowed them to exchange points of view and interpretations about crew functioning and performance variations ».
} 
enseignants. Trohel (2005, p. 242), à partir d'une étude de la dynamique des interactions tuteurs-stagiaires confrontant entretien d'auto-confrontation et analyse conversationnelle, propose d'optimiser la relation de tutorat au moyen d'une «formation spécifique des tuteurs, de l'apport d'un superviseur universitaire et du développement de partenariat entre stagiaires ».

\section{$\mathrm{V}$ - CONCLUSION}

La tendance à l'articulation de matériaux empiriques hétérogènes, respectivement qualifiés de données en première et en troisième personnes, revêt, dans le champ des Sciences du sport, une tournure inédite, notamment sous l'impulsion de chercheurs en STAPS engagés dans le programme du Cours d'action ${ }^{39}$. Ils opérationnalisent empiriquement, à propos d'actions sportives, l'objet théorique du cours d'in-formation (Sève et al., 2012). L'enjeu affiché est de dépasser le caractère insuffisant de la prise en compte exclusive des descriptions phénoménologiques, au moyen de leur confrontation avec des matériaux objectivables. ${ }^{40}$ L'articulation des points de vue extrinsèques et intrinsèques est ici un outil méthodologique au service de la fin que constitue l'objet théorique du cours d'in-formation visant l'accès à la globalité et à la complexité des actions. Elle lui fournit un observatoire singulier, inédit, en complément de ceux déjà largement opérationnalisés empiriquement.

Le programme de recherche du Cours d'action, comme tout programme, doit alors être vu comme une dynamique vivante qui, tout en maintenant intact un «noyau dur » irréfutable ${ }^{41}$ (Lakatos, 1994 ; Theureau, 2009), développe successivement, dans sa ceinture de protection, des hypothèses auxiliaires, analytiques et synthétiques, instables, ou plus précisément des objets théoriques divers et révisables pour tendre au maximum ${ }^{42}$ vers les exigences épistémiques et ontologiques du noyau dur et dépasser certaines frustrations et insatisfactions (Sève et al., 2012).

Au-delà de la dynamique intrinsèque de développement du programme du Cours d'action, d'autres circonstances, contingentes, peuvent expliquer ce

\footnotetext{
${ }^{39}$ Et dans une moindre mesure dans la psycho-phénoménologie.

${ }^{40} \mathrm{La}$ frustration inverse est également possible, comme chez Récopé (2007, p 50) insatisfait d'une prise en compte originelle exclusive, dans ses travaux, des seules données en troisième personne : «j'en étais resté dans ma thèse à un point de vue en troisième personne, certes subjectophile, mais qui me faisait élaborer de l'extérieur un point de vue que je prêtais au sujet indépendamment du point de vue qu'il aurait lui-même pu me donner. J'ai finalement admis la nécessité, mais aussi les difficultés, de procéder, à l'introduction des points de vue en première personne comme ressource complémentaire à l'exploitation des traces et des observables qui caractérisent le point de vue en troisième personne ».

${ }^{41}$ L'activité humaine est considérée, dans le cadre du Cours d'action, comme une totalité émergente, située, incarnée, autonome, dynamique; elle donne lieu à expérience; le couplage structurel entre l'acteur et son monde est tenu pour asymétrique (Saury et al., 2010; Gal-Petitfaux et al., 2013; Bourbousson \& Fortes, 2012).

${ }^{42}$ Tout objet théorique est une réduction méthodologique assumée, ne prétendant pas rendre compte de l'ensemble des niveaux d'organisation de l'activité (Saury et al., 2010) mais permettant une «description symbolique acceptable» de la dynamique du couplage structurel d'un acteur avec sa situation. Le cours d'in-formation doit être vu comme un pas supplémentaire pour saisir la diversité des niveaux de structuration de l'action, leur complexité et leur interaction. Il demeure toutefois une réduction.
} 
souci d'articulation des matériaux hétérogènes. Parmi celles-ci, notons le profil singulier des chercheurs articulatoires ${ }^{43}$, le mouvement de réhabilitation de la subjectivité de l'acteur et de son expérience vécue ainsi que la sensibilité symbolique contemporaine ${ }^{44}$ à la thématique de la conciliation des contraires (Quidu, 2012a). La genèse de travaux scientifiques articulant des données hétérogènes peut également être lue comme une actualisation concrète d'un mouvement d'ensemble plus vaste d'articulation de la pluralité paradigmatique (Quidu, 2012a) et plus fondamentalement d'articulation de la pluralité tout court $^{45}$.

Qu'il réponde à un mouvement d'ensemble contingent ou à une dynamique intrinsèque au programme, le développement des études articulant des matériaux hétérogènes est en cours. La présente réflexion épistémologique a analysé ici l'émergence sur le vif, en train de se faire, in process d'une méthodologie originale ${ }^{46}$. Dans le cadre de l'activité de science normale (Kuhn, 1983) en voie d'installation, de nouveaux objets d'investigation vont être progressivement conquis. Parmi ceux-ci, mentionnons la possibilité d'étudier les stratégies perceptives des sportifs, en combinant l'étude de leurs mouvements oculaires (données en troisième personne via des techniques d'oculométrie objectivant les focalisations fovéales) et de leurs fenêtres attentionnelles (données en première personne via des entretiens d'explicitation renseignant les perceptions périphériques). Pourrait également être envisagée une étude sur les divers modes, émergent versus évènementiel, de timing (i.e. gestion temporelle du mouvement) confrontant d'un côté des analyses fractales des séries temporelles de tapping (voir Lemoine, 2007) pour les données en troisième personne) et de l'autre une analyse des stratégies conscientes de régulation temporelle et des ressentis associés (données en première personne à construire).

D'autre part, l'objet théorique du cours d'in-formation, formulé originellement de façon spéculative par Theureau et dont les premières tentatives d'opérationnalisation empirique ont porté sur les actions sportives, n'a pas vocation à se restreindre au champ des Sciences du sport ${ }^{47}$. Le

\footnotetext{
${ }^{43}$ Ceux-ci reconnaissent une sensibilité ontologique pour la complexité et la complémentarité, possèdent une expérience antérieure d'enseignant d'EPS, manifestent un souci de l'utilité pratique des connaissances académiques...

${ }^{44}$ Voir Quidu (2012a) sur l'idée d'une contestation contemporaine de l'imaginaire diurne « schizomorphe » qui sépare, oppose et disjoint au profit d'un imaginaire nocturne, «synthétique », qui relie (Durand, 1968). Il pourrait s'agir d'un véritable «bassin sémantique », c'est-à-dire d'une réorientation symbolique globale affectant un large spectre d'activités humaines (artistiques, économiques...).

${ }^{45}$ Voir Quidu (2012c) sur la tendance contemporaine à l'articulation de la pluralité non épistémique (axiologique, juridique...). Voir aussi Latour (1991) et Andrieu (2008) sur la prolifération des hybrides ou Delalandre \& Quidu (2015) sur le développement d'une pratique sportive hybride, le mixed martial arts.

${ }^{46}$ Cette saisie d'un programme sur le vif contraste avec l'opinion de Bachelard sur les philosophes des sciences qu'il accuse d' « être en retard d'une mutation de l'intelligence scientifique », de « travailler sur des problèmes scientifiques périmés » (Canguilhem, 1968).

${ }^{47} \mathrm{Ce}$ dernier a constitué un laboratoire favorable à l'expérimentation empirique sur le cours d'information, notamment de par sa structure institutionnelle intrinsèquement pluridisciplinaire, à la
} 
programme du Cours d'action qui l'a développé est issu de l'ergonomie ${ }^{48}$ et sera donc susceptible d'appliquer ce nouvel objet théorique à toutes les activités au travail, dans des domaines aussi variés que la création artistique (musicale ou chorégraphique), l'innovation technologique ou l'enseignement. Dans un sens, les contributions de Saury, Sève, Seifert, Gal-Petifaux et leurs collaborateurs peuvent être considérées comme des travaux empiriques précurseurs susceptibles de fournir des exemples en acte à des études abordant d'autres champs de l'activité humaine.

Si l'effort d'articulation des données hétérogènes ne se résume pas au champ des Sciences du sport, il ne se limite pas non plus, dans le cadre des Sciences cognitives, au programme du Cours d'action. Un autre programme de recherche, la neuro-phénoménologie $e^{49}$, partage cette exigence. Celle-ci confronte des enregistrements de l'activité neuro-électrique du cerveau obtenus via des techniques d'imagerie (troisième personne) et des récits psychophénoménologiques d'expériences vécues obtenus via l'entretien d'explicitation (première personne). Il est né d'une critique des modélisations «éliminativistes » en sciences cognitives (Andrieu, 2007; Quidu, 2011b). En contre-point, Varela milite pour une «réhabilitation de l'expérience vécue (laquelle nécessite une méthode disciplinée d'accès) dans l'étude de la cognition » au travers du concept d'énaction postulant le «double sens de la corporéité, structure à la fois physique et vécue, biologique et phénoménologique ».

Dans le cadre du programme neuro-phénoménologique, nous pouvons retrouver les diverses modalités articulatoires identifiées ci-avant. En effet, la confrontation des données en première et troisième personnes a tout d'abord permis de renforcer la robustesse de certains résultats par triangulation. Petitmengin (2006) a ainsi pu corroborer (ou valider) à partir d'une description fine de la microstructure dynamique de l'expérience vécue, la thèse énactive d'un avènement conjoint du soi et $\mathrm{du}$ monde, laquelle thèse avait été originellement démontrée au moyen de données objectivables en troisième personne. Ont également pu être révélées des corrélations entre d'un côté le récit d'une expérience vécue et de l'autre les synchronisations neurales. Petitmengin (2005) a, dans cette perspective, démontré qu'à des modifications de la dynamique neuronale dans la période précédant une crise d'épilepsie correspondaient des modifications de l'expérience subjective (sentiment de malaise). S'instaure, dans d'autres $\operatorname{cas}^{50}$, un réseau de contraintes génératives mutuelles: ici, les analyses neurodynamiques et phénodynamiques se guident, se déterminent et s'enrichissent mutuellement (fonction heuristique). Plus précisément, «la découverte d'une régularité sur un plan suscite celle d'une

différence par exemple de l'ergonomie, marquée par le monopole de la psychologie, et donc moins à même d'apprécier les contraintes et effets extrinsèques sur les cours d'expérience (Sève et al., 2012).

${ }^{48}$ L'ambition originelle du programme résidait dans l'étude des pratiques « réelles » de travail telles qu'elles sont vécues en situation par les acteurs, au-delà de toute restriction de l'analyse à la dimension " prescrite » des tâches.

${ }^{49}$ Voir Varela, Thompson \& Rosch (1993) ou Varela (1996) pour la philosophie générale.

${ }^{50}$ Les diverses modalités articulatoires ne sont pas cloisonnées : ainsi, chez Petitmengin (2005), le projet originel de recherche de corrélations a progressivement dérivé vers l'instauration d'un réseau de contraintes génératives mutuelles. 
régularité, insoupçonnée, sur l'autre plan en se constituant en critère d'analyse » (Peschard, 2004). ${ }^{51}$ Enfin, à l'instar du Cours d'action appliqué à la motricité sportive, la neuro-phénoménologie est finalisée par des préoccupations pratiques, des visées transformatives, en l'occurrence thérapeutiques. Ainsi, pour Petitmengin (2005, p. 64), « la circulation neurophénoménologique permet d'affermir les bases d'une thérapie cognitive, non pharmacologique, de l'épilepsie ».

L'enjeu demeure in fine de tenir à distance toute menace de réduction, voire d'élimination, que celle-ci soit opérée depuis le pôle des données en première personne (il s'agirait ici d'une réduction «mentaliste » par la phénoménologie) ou depuis celui des données en troisième personne (réduction « matérialiste $\aleph^{52}$ ). Pour Petitmengin $(2005$, p. 64), «la découverte d'homomorphismes entre les structures neurodynamiques et phénodynamiques permet de créer des passerelles entre les deux rives du gouffre qui sépare l'expérience subjective de l'activité neurophysiologique, sans pour autant réduire l'une à l'autre ». Quant à Bitbol (2006), il refuse de voir dans le travail fondateur de Varela la thèse d'une "primauté ontologique de l'expérience vécue » ou une quelconque réactualisation de la théorie de l'identité entre processus psychiques et neurologiques. À l'inverse, «ce que [Varela] fait valoir est l'intérêt pour la science ${ }^{53}$ d'une pratique de prise en compte de la totalité des aspects de cette expérience, qu'ils soient subjectifs ou objectifs » (p. 135). Dit autrement, toujours selon Bitbol (p. 135), Varela «n'affirme pas une identité neuro-mentale, mais vise à instituer une relation étroite entre les deux modalités d'investigation, neuronale et expérientielle ». Au final, une « science de la conscience équitable » est davantage une stratégie de recherche que l'on pratique (et les Sciences du sport y ont apporté une notable contribution) qu'une affirmation ontologique fondationnaliste que l'on récite; en d'autres termes, il s'agit d'une démarche, voire d'une posture méthodologique plus que d'une théorie.

\section{RÉFÉRENCES}

Andrieu, B. (2007). La neurophilosophie. Paris, Presses Universitaires de France. Andrieu, B. (2008). Devenir hybride. Nancy, Presses Universitaires de Nancy.

\footnotetext{
${ }^{51}$ Par exemple, dans l'étude sur l'anticipation des crises d'épilepsie, «l'investigation de la dynamique de l'expérience vécue ouvre de nouvelles pistes pour l'analyse neurodynamique. Elle suggère de rechercher le corrélat neurologique des sensations anticipatrices ressenties longtemps avant la crise, donc d'analyser les synchronisations de l'EEG à plus grande distance des crises ». Dans le cadre de son étude sur l'émergence d'une perception visuelle en trois dimensions, Lutz (2002) utilise les diverses valeurs d'une catégorie expérientielle (i.e. l'état de préparation du sujet au moment de l'apparition de l'image en termes d'émotion, d'attention, d'anticipation) pour répartir les enregistrements neuroélectriques en trois classes en identifiant trois signatures neuronales dynamiques distinctes (voir Petitmengin, 2005, p. 86).

${ }^{52}$ Dans la théorie éliminativiste, «les concepts mentalistes sont purement et simplement écartés au profit de concepts neurophysiologiques (ce qui évite, moyennant une solution radicale, le problème de leur mise en correspondance) » (Bitbol, 2006, p. 137).

${ }^{53}$ Selon Bitbol (2006), le principe méthodologique, qui est au cœur de la démarche neurophénoménologique, peut être considéré comme universellement pertinent, y compris dans les domaines de la biologie évolutive et de la physique quantique.
} 
Berthelot, J.-M. (1990). L'intelligence du social. Paris, Presses Universitaires de France.

Berthelot, J.-M. (2001). Programmes, paradigmes, disciplines. In J.-M. Berthelot (éd.) Épistémologie des sciences sociales (pp. 457-520). Paris, Presses Universitaires de France.

Bitbol, M. (2006). Une science de la conscience équitable. L'actualité de la neurophénoménologie de Francisco Varela. Intellectica, 43, 135-157.

Bourbousson, J., Cogé G. \& R'Kiouak, M. (2014). Émergence et causalité descendante dans l'activité collective. In M. Quidu (éd.). Innovations théoriques en STAPS et implications pratiques en EPS, Les Sciences du sport en mouvement (pp. 133-158). Paris, L'Harmattan.

Bühlmann, F. \& Tettamanti, M. (2007). Le statut de l'approche qualitative dans des projets de recherche interdisciplinaire. Recherches qualitatives, HS 3, 191-213.

Canguilhem, G. (1968). Étude d'histoire et de philosophie des sciences concernant les vivants et la vie. Paris, Vrin.

Chalabaev, A. (2006). L'influence des stéréotypes sexués sur la performance et la motivation en sport et en EPS. Thèse de doctorat. Université Grenoble I.

Chalabaev, A., Major, B., Cury, F. \& Sarrazin, P. (2009). Physiological Markers of Challenge and Threat Mediate the Effect of Performance-Based Goals on Performance. Journal of Experimental Social Psychology, 45, 4, 991-994.

Collinet, C. (2003). La recherche en STAPS. Paris, Presses Universitaires de France.

Delalandre, M. \& Quidu, M. (2015). Arts martiaux mixtes. In B. Andrieu (éd.). Vocabulaire de philosophie du sport. Rouen, Presses universitaires de Rouen-Le Havre.

Depraz, N. (2004). Le tournant pratique de la phénoménologie. Revue philosophique de la France et de l'étranger, 129, 149-165.

Dumouchel, P. (1999). Émotions : essai sur le corps et le social. Paris, Synthelabo.

Durand, G. (1968). Les structures anthropologiques de l'imaginaire. Paris, Dunod.

Durand, M. \& Arzel, G. (2002). Commande et autonomie dans la conception des apprentissages scolaires, de l'enseignement et de la formation des enseignants. In M. Carbonneau \& M. Tardif (éds.). Les réformes en éducation, leurs impacts sur l'école. Sherbrooke, Éditions. du CRP.

Ericsson, A. \& Simon, H. (1980). Verbal reports as data. Psychological Review, 8, $215-$ 251.

Feyerabend, P. (1979). Contre la méthode. Paris, Seuil.

Fortes, M. (2003). La dynamique de l'estime de soi et du soi physique. Thèse de doctorat. Université Montpellier I.

Glaser, B. \& Strauss, A. (1995). La production de la théorie à partir des données. Enquête, 1, 1983-195.

Gouju, J.-L., Vermersch, P. \& Bouthier, D. (2003). Objectivation des actions athlétiques par entretien d'explicitation. STAPS, 62, 59-73.

Grison, B. \& Riff, J. (2002). Validité écologique et situations d'étude privilégiées. Actes des $4^{\text {èmes }}$ journées d'études de l'Association ACT'ING.

Holton, G. (1981). L'imagination scientifique. Paris, Gallimard.

Kuhn T. (1983). La structure des révolutions scientifiques. Paris, Flammarion.

Lahire, B. (1996). La variation des contextes en sciences sociales. Annales, histoire, sciences sociales, 51(2), 381-407.

Lahire, B. (1999). Pour une didactique sociologique. Éducation et sociétés, 4, 29-56.

Lakatos I. (1994). Histoire et méthodologie des sciences. Paris, Presses Universitaires de France.

Latour, B. (1991). Nous n'avons jamais été modernes. Paris, La découverte.

Lemoine, L. (2007). Implication des processus de timing évènementiels et émergents dans la gestion des aspects temporels du mouvement. Thèse de doctorat. Université Montpellier I. 
Lutz, A., Lachaux, J.-P., Martinerie, J. \& Varela F. (2002). Guiding the Study of Brain Dynamics by Using First-Person Data: Synchrony Patterns Correlate with Ongoing Conscious States During a Simple Visual Task. Proc Nat Acad Sci USA, 5, 99(3), $1586-1591$

Lutz, A. (2002). Pour une approche neurophénoménologique des bases neurodynamiques de l'expérience consciente. Thèse de doctorat. Université Paris VI.

Morin, E. (1991). La méthode, tome IV, Les idées. Paris, Seuil.

Peschard, I. (2004). La réalité sans représentation. Thèse de doctorat. École polytechnique.

Petitmengin, C. (2005). Un exemple de recherche neuro-phénoménologique: l'anticipation des crises d'épilepsie. Intellectica, 40, 63-89.

Petitmengin, C. (2006). L'énaction comme expérience vécue. Intellectica, 43, 85-92.

Quidu, M. (2009). Les thêmata dans la recherche en STAPS. STAPS, 84, 7-25.

Quidu, M. (2011a). Les STAPS face à la pluralité épistémique. Thèse de doctorat, Université Nancy II.

Quidu, M. (2011b). L'aventure du corps dans l'épistémologie au $20^{\text {ème }}$ siècle. In B. Andrieu (éd.). Le corps du chercheur. Nancy, Presses Universitaires de Nancy.

Quidu, M. (2012a). Les résonances symboliques des innovations paradigmatiques contemporaines. In M. Quidu (éd.). Les sciences du sport en mouvement. Paris, L'Harmattan.

Quidu, M. (2012b). Approches pragmatique et dispositionnaliste du rapport aux savoirs scientifiques des enseignants d'éducation physique, agrégés et normaliens. Revue des sciences de l'éducation, 38, 1, 161-186.

Quidu, M. (2012c). Traiter la pluralité en science et en société : quelles homologies ? Congrès de la Société de philosophie des sciences. Montréal, 1-3 juin 2012.

Quidu, M. (éd.) (2014). Épistémologie du corps savant: tome I, Le chercheur et la description scientifique du réel. Paris, L'Harmattan.

Schön, D. (1994). Le praticien réflexif. Québec, Logiques.

Sève ,C., Theureau, J., Saury, J. \& Haradji, Y. (2012). Drôles d'endroits pour une rencontre : STAPS, ergonomie et Cours d'action. In M. Quidu (éd.). Les Sciences $d u$ sport en mouvement. Paris, L'Harmattan.

Soler, L., Trizio, E., Nickles, T. \& Wimsatt, W. (2012). Characterizing the Robustness of Science after the Practice Turn in Philosophy of Science. Dordrecht (Netherlands),, Springer.

Soler, L. (2014). Pluralité des paradigmes et dimensions de l'incommensurabilité en physique. In M. Quidu (éd.). Innovations théoriques en STAPS et implications pratiques en EPS, Les Sciences du sport en mouvement. Paris, L'Harmattan.

Terral, P. (2003). Sociologie des conceptions épistémiques. Thèse de doctorat. Université Paris IV.

Theureau, J. (2004). Le Cours d'action : méthode élémentaire. Toulouse, Octares.

Theureau, J. (2009). Le Cours d'action : méthode réfléchie. Toulouse, Octares.

Theureau, J. (2010). Les entretiens d'auto-confrontation et de remise en situation par les traces matérielles et le programme de recherche «cours d'action». Revue d'anthropologie des connaissances, 4/2, 287-322.

Van Der Maren, J-M. (1996). La recherche qualitative peut-elle être rigoureuse ? Cahier de recherche HEC, 96.

Vanhelst, J., Béghin, L., Bui-Xuân, G. \& Mikulovic, J. (2012). Évaluation de l'activité physique chez l'enfant en surcharge pondérale après un programme de réadaptation : le questionnaire contre l'accélérométrie. STAPS, 95, 67-73.

Varela, F., Thompson, E. \& Rosch, E. (1993). L'inscription corporelle de l'esprit. Paris, Seuil.

Varela, F. (1996). Neurophenomenology: a Methodological Remedy for the Hard Problem. Journal of Consciousness Studies, 3, 330-349. 
Vermersch, P. (1994). L'entretien d'explicitation en formation continue et initiale. Paris : ESF.

Vermersch, P. (2000). Conscience directe et conscience réfléchie. Intellectica, 31, 269311.

Vermersch, P. (2010). Les points de vue en première, seconde et troisième personnes dans les trois étapes d'une recherche : conception, réalisation, analyse. Expliciter, $85,19-32$.

Wimsatt, W. (1981). Robustness, Reliability and Overdetermination. In M. Brewer \& B. Collins (eds.). Scientific Inquiry and the Social Sciences (pp. 124-163). San Francisco, Jossey-Bass.

\section{ANNEXE I : CORPUS EMPIRIQUE EN SCIENCES DU SPORT.}

Berteloot, A. (2008). La perception de l'effort chez des coureurs de demi-fond en compétition. Mémoire de Master. Université Rennes II.

Bourbousson, J. \& Fortes, M. (2012). Anthropologie cognitive et théorie des systèmes dynamiques : quelles articulations possibles dans l'analyse de l'activité collective ? In M. Quidu (éd.). Les Sciences du sport en mouvement (pp. 171-185). Paris, L'Harmattan.

Burns, A-M. (2013). On the Relevance of Using Virtual Humans for Motor Skills Teaching: a Case Study on Karate Gestures. Thèse de doctorat. Université Rennes II.

Colin J. (2011). Analyse de l'intervention d'un enseignant d'EPS auprès d'handicapés mentaux en classe ULIS et en classe de $6^{\text {ème }}$. Mémoire de Master. Université Rennes II.

Gal-Petitfaux, N., Adé, D., Poizat, G. \& Seifert, L. (2013). L’intégration de données biomécaniques et d'expérience pour enrichir la compréhension de l'activité et favoriser la conception d'un dispositif technologique : l'exemple d'une étude chez des nageurs de haut niveau. Le travail humain, 76, 257-282.

Martinent, G., Ledos, S. \& Nicolas, M. (2014). Méthodologies à la $1^{\text {ère }}$ et à la $3^{\text {ème }}$ personnes. Quelles articulations possibles dans le champ de la psychologie des émotions en contexte compétitif ? In M. Quidu (éd.). Innovations théoriques en STAPS et implications pratiques en EPS, Les Sciences du sport en mouvement (pp. 291-306). Paris, L'Harmattan.

Mouchet, A. (2003). Caractérisation de la subjectivité dans les décisions tactiques des joueurs d'élite 1 en rugby. Thèse de doctorat. Université Bordeaux 2.

Mouchet, A. (2012). Articulation des modèles scientifiques pour éclairer la complexité des décisions en sport. In M. Quidu (éd.). Les Sciences du sport en mouvement (pp. 243-268). Paris, L'Harmattan.

Mouchet, A. (2014). Implications pédagogiques de l'approche psychophénoménologique en STAPS. In M. Quidu (éd.). Innovations théoriques en STAPS et implications pratiques en EPS, Les Sciences du sport en mouvement. (pp. 529552). Paris, L'Harmattan.

Nordez, A. (2006). Caractérisation et modélisation du comportement mécanique du complexe musculo-articulaire en conditions passives. Thèse de doctorat. Université de Nantes.

Poizat, G. (2006). Analyse en ergonomie cognitive de l'activité collective en tennis de table. Thèse de doctorat. Université de Rouen.

Poizat, G., Bourbousson ,J., Saury, J. \& Sève, C. (2009). Analysis of Contextual Information Sharing during Table Tennis Matches: An empirical study on coordination in sports. International Journal of Sport \& Exercise Psychology, 7, 465-487.

Poizat, G., Poupommeau, C., Seifert, L. \& Haradji, Y. (2011). Analyser l'activité de glaciéristes pour concevoir des piolets en termes d'appropriation. In C. Sève \& 
G. Poizat (éds.), Analyse de l'activité et conception en sport. $14^{\text {ème }}$ Congrès de l'ACAPS.

Poizat, G., Haradji, Y. \& Seifert, L. (2011). Concevoir du matériel sportif à partir d'une approche centrée sur l'activité : une alternative en ergonomie du sport. STAPS, 94, 71-83.

Poizat, G., Bourbousson, J., Saury, J. \& Sève, C. (2012). Understanding Team Coordination in Doubles Table Tennis: Joint Analysis of First- and Third-Person Data. Psychology of Sport and Exercise, 13, 5, 630-639.

Récopé, M. (2007). Normativité et sensibilité : une perspective généalogique d'étude du mouvement et de l'action. HDR. Université Clermont-Ferrand.

Ria, L. (2006). L'entrée dans le métier des enseignants du second degré. HDR. Université Clermont-Ferrand.

Roberts, J. R., Jones, R. \& Rothberg, S. J. (2001). Measurement of Contact Time in Short Duration Sports Ball Impacts: an Experimental Method and Correlation with the Perceptions of Elite Golfers. Sports Engineering, 4, 191-203.

Roberts, J.R., Jones, R., Rothberg, S.J., Mansfield, N.J. \& Meyer, C. (2006). The Influence of Sound and Vibration from Sports Impacts on Players' Perceptions of Equipment Quality. Proceedings of the Institution of Mechanical Engineers, 220, 215-227.

Saury, J., Nordez, A. \& Sève, C. (2010). Coordination interindividuelle et performance en aviron : apports d'une analyse conjointe du cours d'expérience des rameurs et de paramètres mécaniques. Activités, 7, 2-27.

Seifert, L. (2010). Coordination motrice et expertise : Pour une approche complexe du contrôle moteur dans les activités physiques et sportives. HDR. Université Rouen.

Seifert, L., Wattebled, L., Herault, R., Poizat, G., Adé, D., Gal-Petitfaux, N. \& Davids, K. (2014). Neurobiological Degeneracy and Affordance Perception Support Functional Intra-Individual Variability of Inter-Limb Coordination during Ice Climbing. PlosOne, 9(2).

Sève, C., Nordez, A., Poizat, G. \& Saury, J. (2013). Performance analysis in sport: Contributions from a joint analysis of athletes' experience and biomechanical indicators. Scand. J. Med. Sci. Sports, 23(5), 576-584.

Trohel, J. (2005). Les interactions tuteur-stagiaire en situation d'entretien de conseil pédagogique au cours de la formation des enseignants d'EPS. Articulation des cours d'action et dynamique de la conversation. Thèse de doctorat. Université Rennes II.

Visioli, J. (2009). Émotions, corps et théâtralité. Contribution à une modélisation de l'expertise quotidienne de l'activité des enseignants en Éducation Physique et Sportive. Thèse de doctorat. Université Rennes II. 